\title{
Les ouvrages de prise et de rejet de la centrale nucléaire de Gravelines
}

PAR

P. Mainier

E.D.F., Direction de l'Équipement
ET

\author{
$\mathbb{J} \circ \sim \mathbb{P}$. Lepetit \\ E.D.F., Laboratoire National d'Hydraulique
}

\section{Les ouvrages de la centrale de Gravelines}

\section{DISPOSITIONS GÉNÉRALES}

La centrale de Gravelines est située en bordure de la mer du Nord entre les villes de Dunkerque et Calais, immédiatement à l'Ouest de la jetée ouest de nouvel avantport de Gravelines (fig. 1).

Rètenu à l'origine pour l'installation de tranches thermiques, le projet a évolué vers un site mixte pouvant comporter 2 tranches thermiques de $700 \mathrm{MW}, 4$ tranches nucléaires à eau pressurisée de $900 \mathrm{MW}$ et 4 tranches nucléaires à eau pressurisée de $1350 \mathrm{MW}$.

Les terrains sur lesquels se trouve la centrale ont été, en grande partie, gagnés sur la mer par remblaiement ( 70 ha environ sur un total de 120) en profitant des sables extraits pour le creusement du nouvel avant-port. Le niveau de la plateforme a été fixé à 8,50 (cote marine) afin de mettre l'ensemble des ouvrages à l'abri des plus hautes 'mers avec une probabilité de l'ordre de 10-6/an. On peut reconstituer, d'après la figure, le tracé de l'ancien rivage qui passe à l'extrémité de la quatrième tranche $900 \mathrm{MW}$.

Le choix d'un site en bord de mer est essentiellement lié au fait que l'on dispose d'une source froide permettant la réfrigération des condenseurs en circuit ouvert.

La consommation d'eau de refroidissement prévue pour le site est de l'ordre de $420 \mathrm{~m}^{3} / \mathrm{s}$ se répartissant grossièrement en $40 \mathrm{~m}^{3}$ pour les 2 tranches thermiques de $700 \mathrm{MW}$, $160 \mathrm{~m}^{3}$ pour les 4 tranches de $900 \mathrm{MW}$ et $220 \mathrm{~m}^{3}$ pour les 4 tranches nucléaires de $1350 \mathrm{MW}$.

Pour éviter au maximum les risques de recyclage d'eau chaude entre le rejet et la prise d'eau, il était naturel de songer à utiliser la grande digue ouest de l'avant-port, afin de séparer les ouvrages de prise et de rejet et, dans ce cas, d'installer la prise d'eau dans l'avant-port lui-même où l'on trouve rapidement des fonds très importants. Dans ces conditions, la distribution de l'eau aux différentes stations de pompage des tranches projetées se fait par un canal parallele aux installations.

En ce qui concerne les rejets, il aurait été possible d'envisager, pour chaque tranche, un ouvrage sur la plage artificielle créée par le remblaiement, mais le souci de conserver pour le public la plus grande longueur de plage possible, nous a conduit à prévoir un ouvrage de rejet unique pour l'ensemble du site accolé à la digue ouest de l'avantport. Un canal de rejet recueillant les eaux réchauffées de chaque tranche vient déboucher dans cet ouvrage.

Quels sont les problèmes posés par une installation de ce type en bordure de mer?

Tout d'abord l'importance des débits : $420 \mathrm{~m}^{3} / \mathrm{s}$. C'est un débit supérieur au débit moyen de la Seine à son embouchure. Ces débits importants entrainent des ouvrages de dimensions peu habituelles jusqu'à ce jour, pouvant être soumis à l'action de la houle et sur lesquels l'influence de la marée a une importance considérable.

Corrélativement à l'étude de ces ouvrages, il a fallu regarder :

- leur influence sur l'hydrologie locale (conditions de navigation dans l'avant-port, conséquences éventuelles sur la jetée ouest);

- les problèmes posés par l'échauffement de $12^{\circ} \mathrm{C}$ de cette grande masse d'eau (dimensions et forme de la tache thermique).

Pour nos études de conception des ouvrages, nous avons eu la chance de bénéficier de celles faites par le Port Autonome de Dunkerque, dont la collaboration nous a été très précieuse étant donnée sa grande expérience pour les travaux à la mer dans la région de Gravelines. 


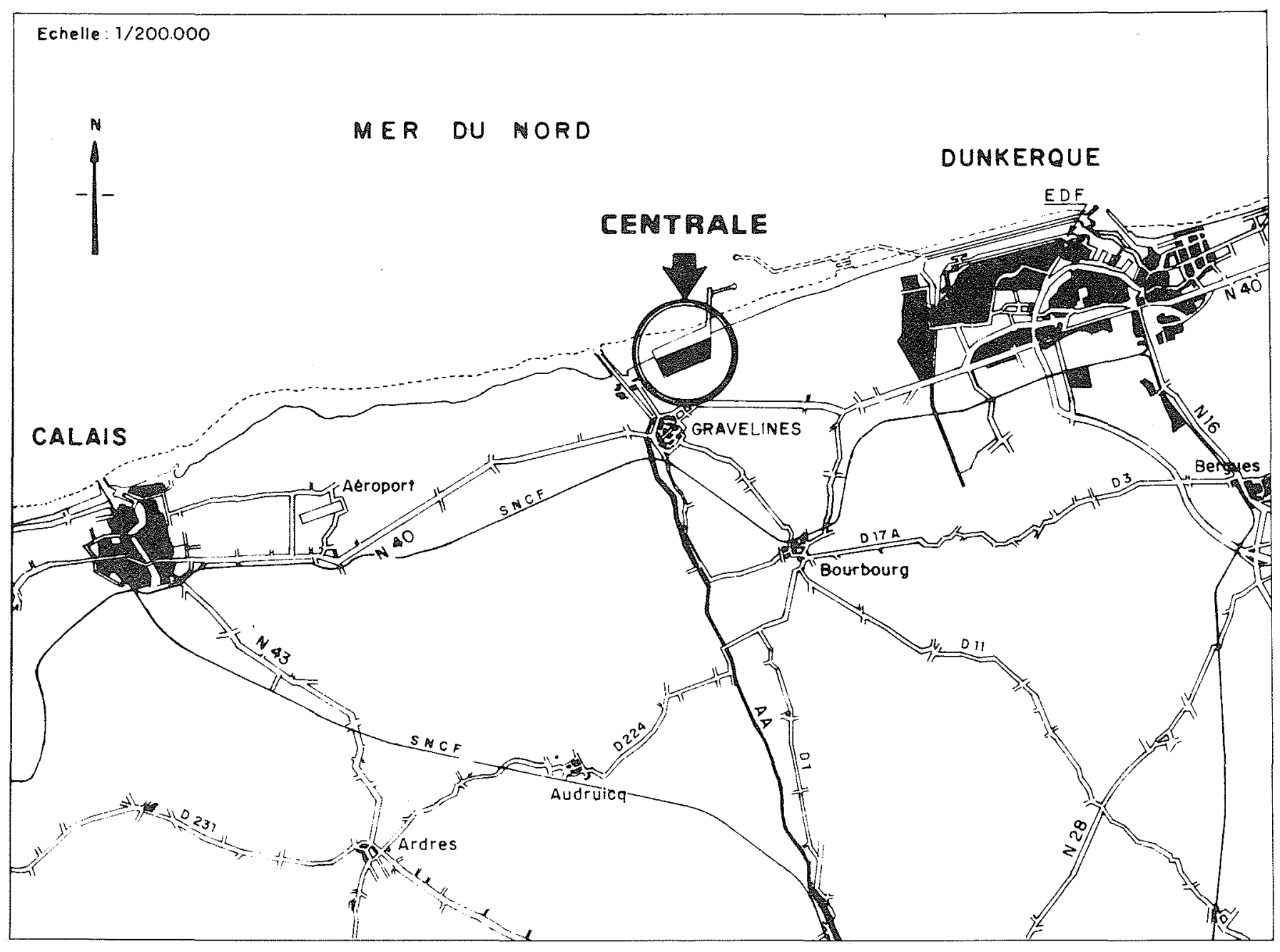

$1 /$ Plan de situation

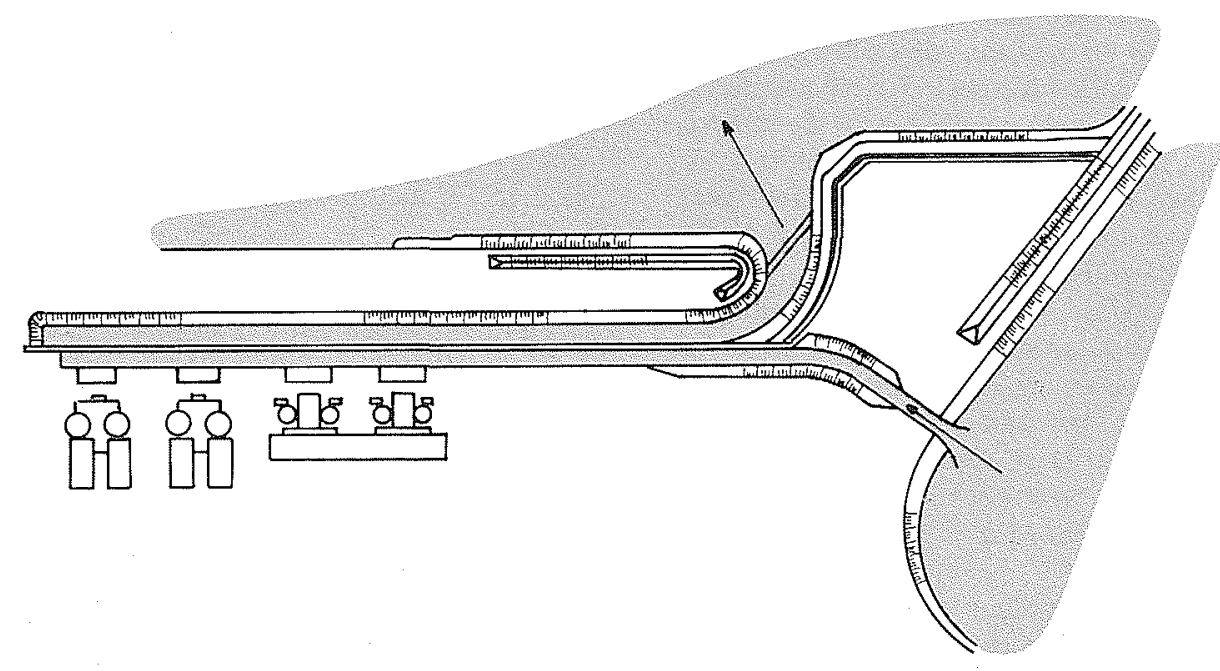

2 / Plan d'ensemble 


\section{L'OUVRAGE DE PRISE D'EAU}

Le canal d'amenée d'eau à la centrale a, dans sa partie courante, une profondeur de $6 \mathrm{~m}$ au-dessous des plus basses eaux et une largeur de $35 \mathrm{~m}$.

Ces dimensions ont été fixées pour qu'à basse mer:

- les risques d'érosion restent faibles et n'entraînent pas des protections trop importantes; la vitesse de l'eau ne dépasse pas $2 \mathrm{~m} / \mathrm{s}$;

- la perte de charge à l'extrémité du canal ne soit pas trop importante (elle est au maximum de l'ordre du mètre).

Comme l'eau est prise dans un très grand avant-port, (fig. 2) dont les fonds sont à $20 \mathrm{~m}$ au-dessous des plus basses mers et où les vitesses dues aux courants de marée restent extrêment faibles, nous n'avons pas eu à nous préoccuper du problème de l'ensablement possible du canal dans les périodes de faible débit et de haute mer (vitesse de l'ordre de $10 \mathrm{~cm} / \mathrm{s}$ ). L'avant-port joue pour la centrale le rôle d'un gigantesque dessableur.

La conception de louvrage de prise résulte des essais menés par le Laboratoire National d'Hydraulique, le but étant de diminuer, dans toute la mesure du possible, les vitesses des courants dans la zone des appontements pétroliers.

Ces essais ont conduit à adopter un convergent bordé par deux murs moulés verticaux, de $80 \mathrm{~cm}$ d'épaisseur, exécutés avant creusement de cette partie de l'avant-port.

L'avant-port étant abrité de la houle et les vitesses restant inférieures évidemment à celles du canal d'amenée, le revêtement du radier est le même que pour celui-ci.

Il est constitué par une couche de $30 \mathrm{~cm}$ d'enrochements tout venant $0 / 150 \mathrm{~kg}$ posés sur un lit de fascines tissées, avec interposition d'une toile de polypropylène jouant le rôle de filtre à sable.

Il faut insister tout particulièrement sur la présence des fascines, qui sont une particularité des travaux dans le port de Dunkerque du fait des moyens développés pour leur mise en cuvre.

Les fascines sont réalisées par des fagots de bois de saule ou de châtaignier, liés tout d'abord en boudin de 5 à $7 \mathrm{~cm}$ de diamètre et rassemblés ensuite, pour constituer un genre de grillage à mailles carrées de dimensions variables. Ce grillage est livré en éléments de $200 \mathrm{~m}$ de long par $15 \mathrm{~m}$ de large.

Une épaisseur de protection comprend en partant du fond:

- une toile de polypropylène de $600 \mathrm{~g} / \mathrm{m}^{2}$;

- un grillage de fascines à mailles de $20 \mathrm{~cm}$;

- une couche de fagots déposés à la main;

— un grillage de fascines à mailles de $1 \mathrm{~m}$.

Les enrochements disposés au-dessus viennent s'interpénétrer dans les fascines, celles-ci évitant leur entraînement.

Au cas où il est nécessaire de poser ce tapis sous l'eau, il suffit d'amener le matelas au-dessus du lieu de pose par flottaison et de l'immerger par chargement au moyen d'enrochements.

Pour l'ouvrage de prise, un renforcement du revêtement du fond par enrochements $1 / 3$ t est prévu au pied des parois moulées sur une épaisseur de $1 \mathrm{~m}$ et une largeur de $5 \mathrm{~m}$.
Le fait de prendre l'eau nécessaire au refroidissement d'une centrale nucléaire dans un avant-port pétrolier a posé deux problèmex importants concernant la sûreté de la centrale.

\section{Il faut en effet éviter :}

I" Que l'échouement d'un navire devant la prise d'eau proprement dite ne risque d'obturer celle-ci, provoquant, si le site est à sa pleine puissance, une baisse rapide des niveaux dans le canal. Des ducs d'Albe constitués par des tubes de $1,80 \mathrm{~m}$ de diamètre à inertie variable disposés en triangle ont été dans ce but placés en avant de la prise.

$2^{\circ} \mathrm{Qu}$ 'à la suite du déchirement de la coque d'un pétrolier, une quantité importante de mazout ne pénètre dans le canal et vienne encrasser les systèmes de réfrigération, surtout ceux touchant la sûreté. Dans ce but, un mur écrèmeur plongeant d'un mètre sous les plus basses eaux est installé près de l'entrée.

\section{L'OUVRAGE DE REJET}

L'ouvrage de rejet proprement dit est le fruit de longues études du Laboratoire National d'Hydraulique, tant du point de vue de sa forme que de sa constitution.

Le canal de rejet a, dans sa partie terminale, une section trapézoïdale avec une largeur de $47 \mathrm{~m}$ au plancher et des parois inclinées à $3 / 1$.

Le fond à la cote 0,00 est constitué de la même manière que celui du canal d'amenée.

Le talus des berges est revêtu:

- entre les cotes 0 et $6 \mathrm{~m}$ (cotes marines), d'une couche d'un mètre d'épaisseur d'enrochements $0 / 500 \mathrm{~kg}$, posés sur une toile de polypropylène;

- entre la cote +6 et le niveau de la plateforme, d'une épaisseur de béton bitumineux de 20 à $40 \mathrm{~cm}$, posé sur une feuille de callendrite.

Au niveau +6 est aménagée une risberme de $3 \mathrm{~m}$ de largeur; celle-ci est bordée d'un rideau de vannage en palplanches.

L'ouvrage de rejet proprement dit est un coude divergent prolongeant le canal.

Un seuil arasé à la cote $1.35 \mathrm{~m}$ a été aménagé dans sa partie centrale, afin d'éviter qu'en période de basse mer l'écoulement dani le canal ne devienne torrentiel (le fond du canal est au niveau des basses mers).

Ce seuil est une digue en enrochements à granulométrie continue $1 / 3 \mathrm{t}$, comportant un noyau central constitué par des palplanches Larsen SL2 de $5 \mathrm{~m}$ de long assurant une étanchéité relative à basse mer.

En aval du seuil, on trouve un radier arasé à la cote 0,00 sur une longueur de $35 \mathrm{~m}$ et rejoignant ensuite la cote $-3,00$ avec une pente de 10/1. Ce radier est constitué par une épaisseur de $1 \mathrm{~m}$ d'enrochements 1 à $3 \mathrm{t}$ déposés sur un lit de fascines.

En raison de leur exposition à la houle frontale, les berges de cet ouvrage sont une succession de talus et de risbermes destinés à provoquer le déferlement des lames.

Jusqu'au niveau +6 , elles sont constituées d'enrochements, 2 à $4 \mathrm{t}$, posés sur un soubassement $0-500 \mathrm{~kg}$, luimême placé sur une toile de polypropylène.

La pente du talus est de $2 / 1$ jusqu'à la cote +5 et $10 / 1$ entre les cotes 5 et 6 . Au-dessus de la cote +6 , on trouve successivement : 


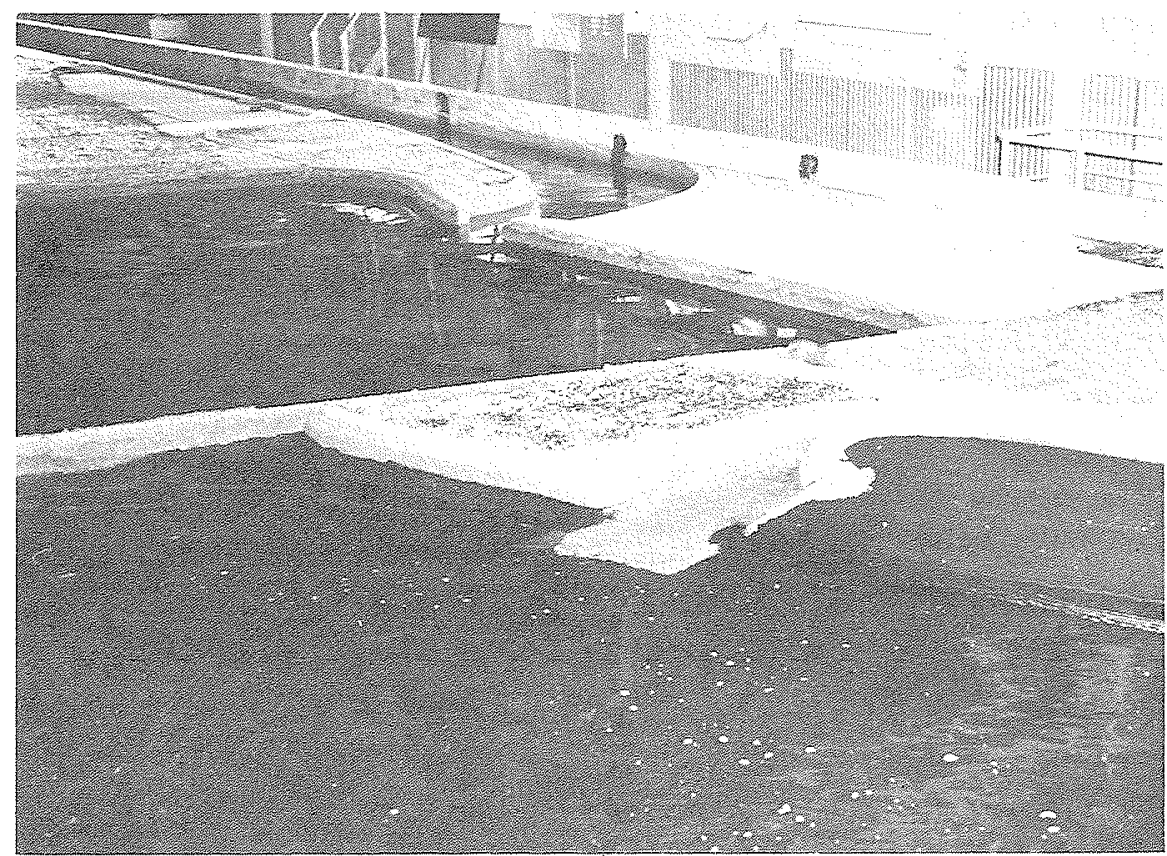

$3 /$ Modèle d'ensemble:

Le canal de rejet à l'ouest de l'avant-port

- un talus incliné à 4 pour 1 ;

- une risberme au niveau $+8,50$;

- un second talus à 4 pour 1 jusquà la cote +12 .

Le tout est recouvert de la couche de béton bitumineux décrite précédemment et déjà largement utilisée par le Port Autonome de Dunkerque.

L'aménagement de cet ouvrage de rejet se fait à sec sous rabattement de nappe, à l'abri d'une digue provisoire en sable, exécutée à l'occasion du remblaiement de la plateforme. Une couche de béton bitumineux la protège contre l'érosion de la houle. Vue en plan, cette digue forme un arc qui vient se raccorder aux berges de part et d'autre de l'ouvrage de rejet, de façon à ce que la zone où le pied de talus n'est pas accessible à marée basse soit tou entière dans l'enceinte provisoire ainsi formée.

Lorsque l'ouvrage de rejet et le canal seront terminés, la couche protectrice en béton bitumineux sera décapée et la digue déblayée hydrauliquement. Entre-temps, les raccordements avec la jetée et la plage à l'extérieur de l'enceinte auront été exécutés à la marée et il suffira de reprendre les talus au droit de la jonction de la digue provisoire avec les berges.

\section{ETUDES DE LABORATOIRE}

Les études de laboratoire ont porté sur quatre points :

- détermination de la tache thermique et du recyclage à la prise;

- fonctionnement hydraulique de la prise;

- fonctionnement hydraulique et sédimentologique du rejet;

- stabilité à la houle des ouvrages de rejet.

Ces études ont eu la chance de bénéficier des études faites de 1967 à 1973 pour la réalisation du nouvel avantport de Dunkerque et des outils mis en ceuvre à cette occasion. On disposait sur le site de nombreuses mesures en nature concernant la houle. les courants de marée. la granulométrie des sédiments et le transport de ceux-ci sous laction de la houle et des courants. Le Laboratoire National d'Hydraulique de Chatou conservait deux modeles réduits opérationnels: lun à fond fixe représentant l'intérieur de l'avant-port (fig. 3) et son voisinage immédiat, l'autre à fond mobile représentant toute la côte et les bancs de sable depuis Dunkerque jusqu'au-delà de Gravelines. Les études de la prise ont été effectuées sur le modèle de l'avant-port et les études du rejet sur le modèle d'ensemble. Ces études ont été complétées par des calculs de tache thermique et par des essais de stabilité à grande échelle en canal.

\section{Tache thermique et recyclage}

La tache thermique a été déterminée par un calcul numérique sur ordinateur, prenant en compte les phénomènes de convection par les courants de marée, de dispersion et d'échange avec l'atmosphère et utilisant les courants de marée mesurés sur le modèle d'ensemble de l'avant-port de Dunkerque. Nous ne développerons pas ici la méthode de calcul, ni les résultats obtenus. mais donnerons seulement des indications sur le rôle joué par l'avant-port de Dunkerque vis-à-vis de ces problèmes (fig. 4).

Les courants de marée parallèles a la côte portent vers l'est à pleine mer et vers louest à basse mer. Le remplissage du port à marée montante et son vidage à marée descendante se produisent donc chacun la moitié du temps avec un courant portant vers l'est et l'autre moitié avec un courant portant vers l'ouest. Le rejet étant situé à l'ouest du port, l'eau chaude ne peut donc parvenir à la prise que pendant la moitié du temps du remplissage du port c'està-dire pendant le quart de la marée.

Les ouvrages du port assurent ainsi un bon isolement entre le rejet et la prise, d'où un recyclage limité. Le calcul montre, en effet, que celui-ci est de l'ordre de $5 \%$ 

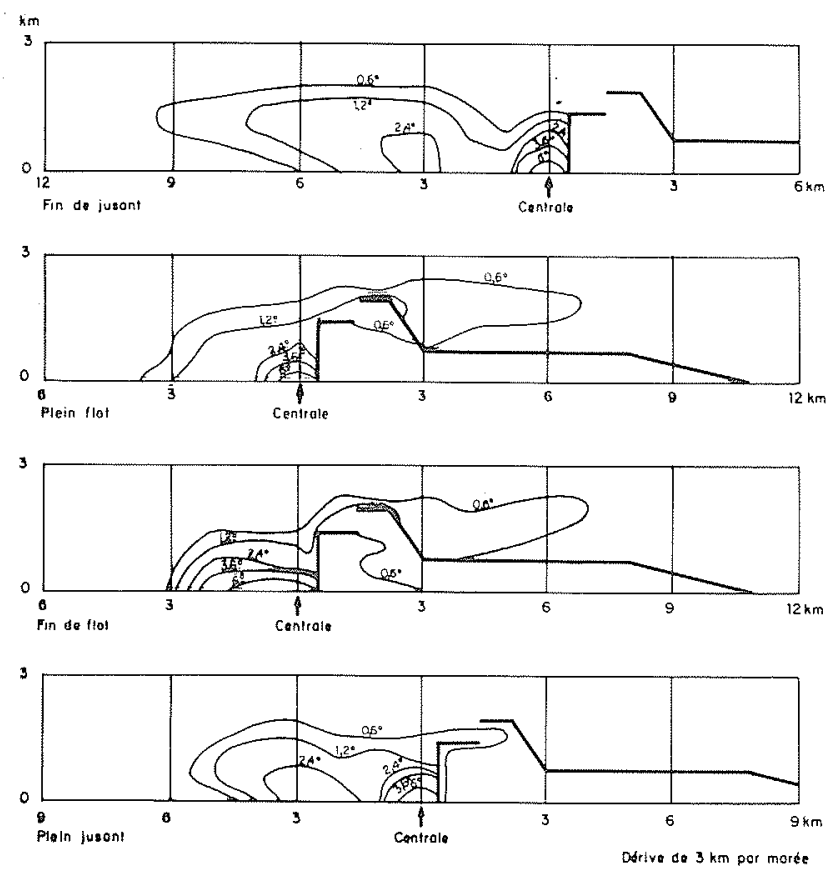

4 / Isothermes pour $4 \mathrm{GW}$ installés

(Déblt : $160 \mathrm{~m}^{3} / \mathrm{s}$, élévation de température : $12{ }^{\circ} \mathrm{C}$ )

pour une puissance installée de $4 \mathrm{GW}$ et de $12 \%$ pour une puissance installée de $10 \mathrm{GW}$. Sous cet aspect, la présence de l'avant-port de Gravelines est donc nettement favorable.

Par contre, l'avant-port qui fait une saillie importante sur la côte modifie l'écoulement des courants de marée et crée, de part et d'autre, une zone d'ombre à courant relativement faible et tourbillonnaire, en particulier le long de la jetée ouest au droit du rejet. Ceci entraîne une moindre dilution de l'eau chaude au voisinage immédiat du rejet, d'où un échauffement des eaux relativement important en cet endroit. Sous cet aspect l'avant-port de Gravelines est donc plutôt défavorable.

Plus au large, l'inflience du port semble être négligeable et la tache thermique a des dimensions comparables à ce qu'elle serait en l'absence de port pour une puissance de $10 \mathrm{GW}$ (échauffement de plus de $1^{\circ} \mathrm{C}$ sur une surface d'environ $20 \mathrm{~km}^{2}$ au lieu de $10 \mathrm{~km}^{2}$ ).

\section{Fonctionnement hydraulique de la prise}

La prise étant située dans l'avant-port, il fallait s'assurer qu'elle ne risquait pas d'induire des vitesses gênantes pour la navigation et l'accostage des navires. En eau calme, une prise fonctionne en puits et les vitesses d'aspiration décroissent comme l'inverse de la distance, c'est-à-dire rapidement. Mais ici l'intérieur de l'avant-port est le siège de vastes tourbillons, dûs au remplissage lors de la marée montante, et l'alimentation de la prise ne se fait pas de façon régulièrement répartie. Des champs de courant ont donc été dressés sur modèle réduit aux différentes heures de la marée (fig. 5); les résultats obtenus mortrent que les vitesses transversales aux navires sont peu augmentées par rapport à ce qu'elles sont sous la seule influence des courąnts de marée.

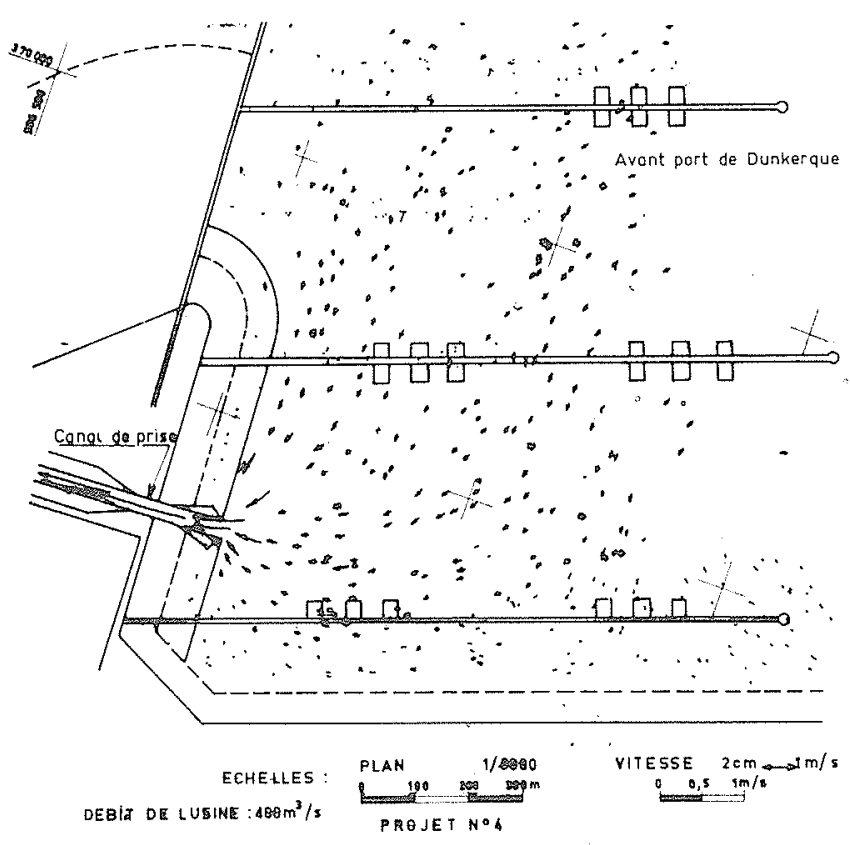

5 / Prise d'eau de la centrale de Gravelines : Champ de courants à $P M-5$

Un deuxième problème abordé est celui de la disjonction des pompes de circulation. Les vitesses dans le canal d'amenée étant élevées, il en résulte des intumescences relativement importantes : la variation totale de cote atteint $1,60 \mathrm{~m}$ en cas de disjonction de l'ensemble de l'usine de $10 \mathrm{GW}$ à basse mer. Celte situation est évidemment fort improbable et en cas de disjonction d'un seul groupe les intumescences sont beaucoup plus faibles.

Lorsque l'avant-port sera terminé, les fonds au large de la prise seront dragués à la cote $-17,50$ et le canal y puisera directement l'eau. En attendant, en phase provisoire, un chenal sera dragué à la cote $-4 \mathrm{~m}$ depuis le cercle d'évitage du port jusqu'à la prise, sur une longueur d'environ $800 \mathrm{~m}$.

Fonctionnement de l'ouvrage de rejet

\section{HYDRAULIQUE}

A basse mer, le débit de $400 \mathrm{~m}^{3} / \mathrm{s}$ se déverse par-dessus le seuil avec une lame d'eau haute de $1,40 \mathrm{~m}$. Les vitesses à la sortie sont alors élevées. A pleine mer, le seuil est complètement noyé et les vitesses de sortie sont beaucoup plus faibles.

\section{SÉdimentologique}

Le principal problème posé par le rejet est de nature sédimentologique. Les premiers essais ont montré que le rejet ne perturbe pas la stabilité générale des fonds au large et, notamment, ne provoque pas d'ensablement du chenal d'accès au port, ni d'érosion le long de la jętée ouest de celui-ci. Mais l'action du rejet se traduit par une érosion très importante immédiatement au large de son débouché. Le débit de $400 \mathrm{~m}^{3}$ est en effet déversé sur une plage de sable fin et il convient de stabiliser cette plage pour éviter une érosion excessive qui pourrait compromet- 


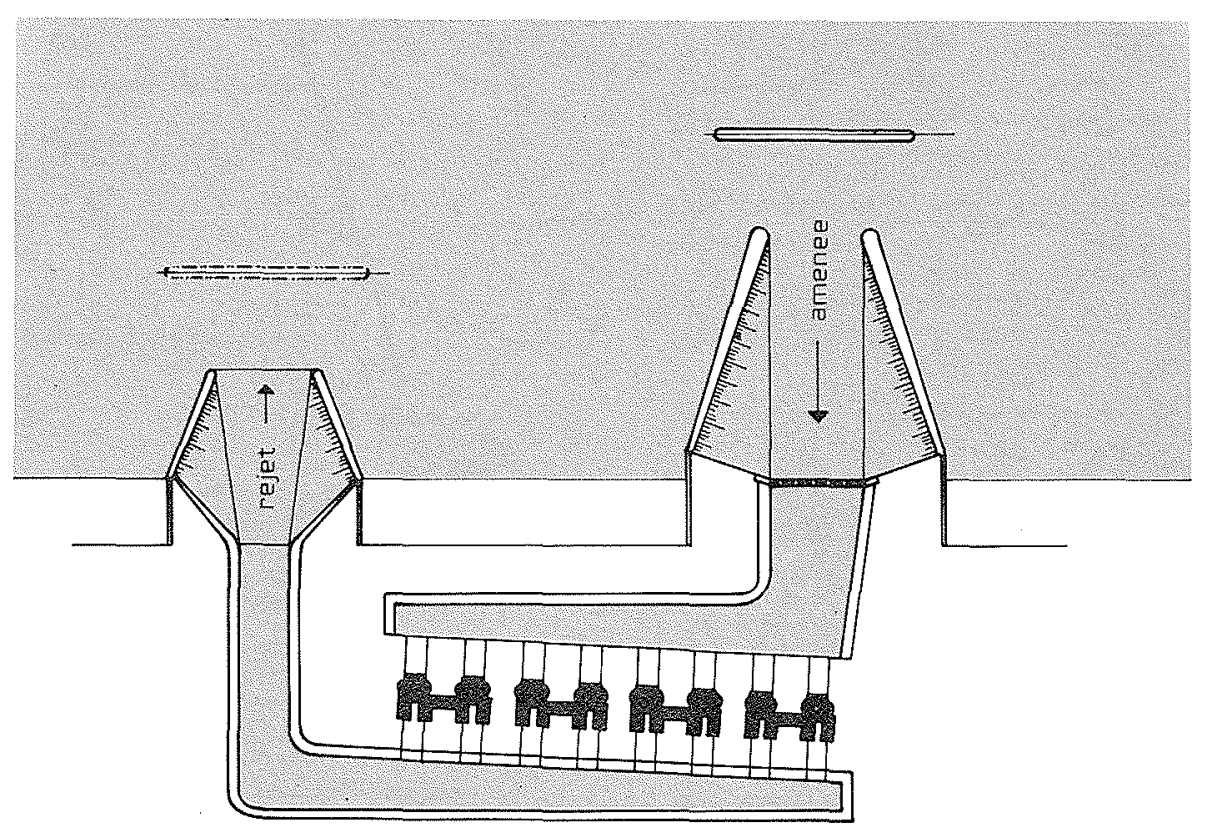

6 / Solution 1

tre la stabilité des ouvrages avoisinants et entrainer une érosion de la plage voisine de Fort-Philippe.

La première solution envisagée consistait à conduire le canal de rejet au-delà de la laisse de basse mer, jusqu'aux fonds de $-3 \mathrm{~m}$, en l'élargissant progressivement jusqu'à une largeur de $250 \mathrm{~m}$ et en stabilisant son fond et ses talus à l'aide d'enrochements. Ce dispositif s'est avéré surdimensionné puisque des dépôts de sable se produisaient dans le canal sur sa moitié ouest.

Un projet beaucoup plus modeste, constitué d'un canal large de $100 \mathrm{~m}$ dragué à la cote -1 et s'étendant uniquement jusqu'au fond de cote +2 , s'est avéré sousdimensionné puisqu'il entraînait un puits d'érosion jusqu'à la cote -9 pour un débit de $400 \mathrm{~m}^{3} / \mathrm{s}$.

La solution retenue après plusieurs essais consiste à ne réaliser que le seuil du canal et une protection réduite au pied du seuil, puis à laisser le chenal se creuser sur la plage sous l'action des faibles débits de rejet qui se produiront lors de la mise en service progressive des premières tranches. L'action, pendant six ans, d'un débit de $150 \mathrm{~m}^{3} / \mathrm{s}$ creuse un chenal large de $200 \mathrm{~m}$ et profond de -2 à $-4 \mathrm{~m}$. Il suffit alors de tapisser le fond de ce chenal avec des enrochements, sur environ $300 \mathrm{~m}$ de longueur, pour assurer une bonne stabilité avec le débit total de $400 \mathrm{~m}^{3} / \mathrm{s}$.

\section{Stabilité à la houle des ouvrages de rejet}

Les études ont été complétées par des essais en canal à houle à grande échelle qui ont permis d'examiner la stabilité des ouvrages en enrochements bordant le canal de rejet de part et d'autre, leur franchissement par la houle et la pénétration de la houle dans le canal au-delà du déversoir. Ces essais ont amené à renforcer la structure des ouvrages dans les coudes et à exhausser le sommet des ouvrages extérieurs; ils ont aussi montré que la houle pénètre peu dans le canal de rejet au-delà du coude.

\section{Ouvrages de prise et de rejet d'eau sur des plages soumises à la marée et ne disposant pas d'installations}

\section{OBJET DES ÉTUDES}

Dans le cas de Gravelines, comme nous l'avons indiqué, l'ensemble des ouvrages d'eau a été élaboré en cherchant à bénéficier de la présence de l'avant-port. E.D.F. a recherché la ou les solutions envisageables dans le cas où elle serait amenée à installer une centrale sur différents types de plage ne bénéficiant d'aucun aménagement antérieur. Les études ont porté sur une cote du "type de Gravelines ", c'est-à-dire une plage de sable fin, avec une pente de l'ordre de 70/1. Elles ont été effectuées en accord entre la Société d'Études et d'Équipements d'Entreprises et E.D.F.

Pour donner plus de corps à ces études, il a fallu fixer certains paramètres n'intervenant que dans l'ampleur des ouvrages et non pas dans leur constitution: le marnage a été pris égal à $8 \mathrm{~m}$ maximum, et le débit appelé à $1000 \mathrm{~m}^{3} / \mathrm{s}$ pour un ensemble de 8 tranches, en circuit ouvert. Le souci de ne pas provoquer de rupture brusque de l'alimentation conduit à des solutions permettant d'avoir en permanence en stockage une quantité d'eau importante. donc un canal d'amenée de dimensions suffisantes. De plus, il est nécessaire d'éloigner les ouvrages de prise et de rejet, de façon à éliminer tout recyclage d'eau entre le rejet et la prise, l'ouvrage de prise devant lui-même se situer au large pour disposer de fonds suffisants.

Différentes solutions ont été examinées, avec des stations de pompage soit en bord de rivage et précédées d'un chenal dragué soit en mer reliées à la Centrale par des conduites posées sur le fond et ensouillées. La grande mo- 


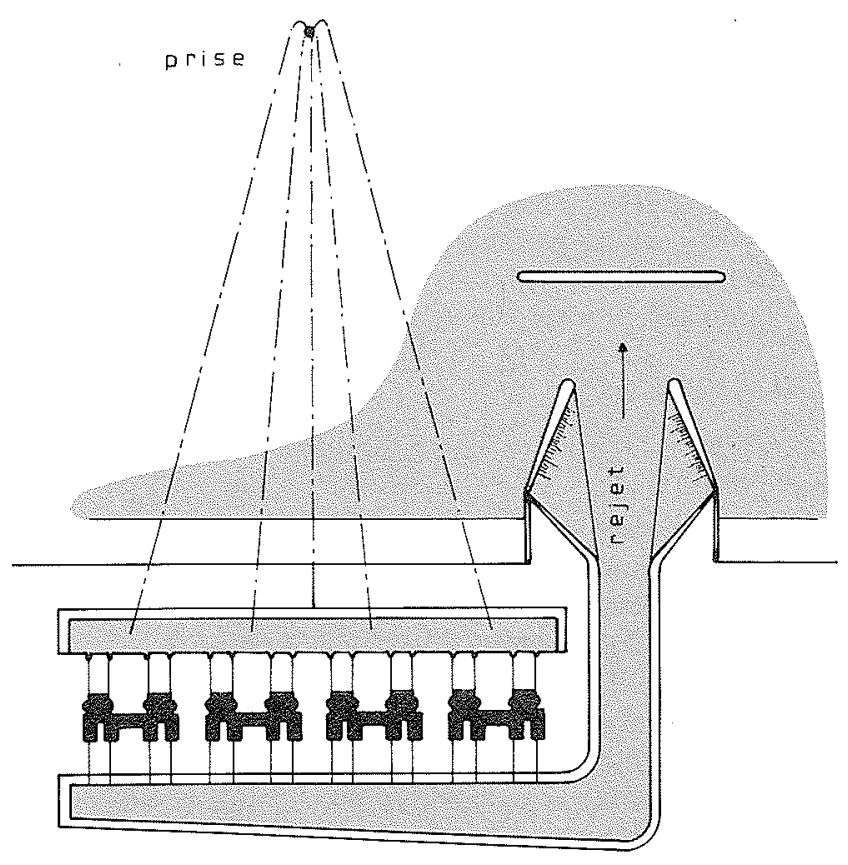

$7 /$ Solution 2

bilité des fonds marins ainsi que les contraintes induites par la houle sur la charge des pompes ont conduit à ne retenir que deux types de solutions:

Solution $l$ : Elle découle directement de la leçon tirée de Gravelines : il s'agit de reconstituer une espèce de port artificiel permettant de prendre l'eau au large en zone calme, sans ouvrage particulier, et de séparer le-rejet de l'amenée. Le rejet est aussi inspiré de Gravelines.

Solution 2: L'eau est prise en haute mer, dans une tour conçue pour amortir la houle, reliée à la centrale par des galeries souterraines. Une variante avec des tours séparées permet l'échelonnement des travaux.

\section{SOLUTION 1}

\section{Description}

Cette solution est représentée figure 6 , avec une distance de la prise d'eau au rivage telle que la cote du fond soit $-5 \mathrm{C} . \mathrm{M}$. à la prise, le talus de la plage étant à $70 / 1$ (pente minimale d'équilibre dans les zones de marnage exposées à la houle, tandis que $30 / 1$ suffisent dans les zones abritées). La prise d'eau est constituée d'un chenal dragué à - 5. large de $400 \mathrm{~m}$, bordé de deux digues en enrochements implantées sur les fonds naturels et reliées au chenal par un lalus non revêtu à 30/1. ce qui explique la forme trapézoïdale de lensemble. Les digues sont du même type que celles de la jetée nord de l'avant-port de Dunkerque. Les talus sont à $4 / 3$, recouverts de blocs de béton de 10 à $20 \mathrm{t}$ du côté exposé à la houle. L'entrée elle-même est protégée par un brise-lame submersible du genre de celui de l'Europort à Rotterdam, dont la nécessité est à étudier pour chaque site.

Le chenal conduit à l'ouvrage de prise proprement dit, constitué d'une succession de piles et de radiers, les piles étant espacées de $15 \mathrm{~m}$. Les piles extrêmes se raccordent sur les digues du chenal au moyen de murs en aile et de rideaux de palplanches. Les piles sont reliées entre elles en partie haute par des écrans, dont le rôle est double :

- ils permettent de prendre l'eau à la profondeur optimale;

- ils constituent un obstacle à la houle résiduelle et aux corps flottants importants.

Vient ensuite le canal d'amenée proprement dit, creusé à la cote -5 , de conception classique, bordé du côté centrale par les stations de pompage. Le canal de rejet, parallèle au canal d'amenée, débouche dans l'ouvrage de rejet, de conception similaire à celui de prise, mais de dimensions moindres, car le chenal n'est dragué qu'à la cote 0 .

\section{Mode d'exécution}

Les terrassements nécessaires à la réalisation des canaux permettent la création, par remblais hydrauliques, d'une plateforme à l'endroit de l'ouvrage de prise d'eau. Cet ouvrage est alors exécuté à $\mathrm{sec}$, de façon classique. Les digues seraient faites à l'avancement, exactement comme pour celles de l'avant-port de Dunkerque: la sous-couche en sable est clapée dans les zones plus profondes que le niveau $-3,50$, les fascines sont mises en place par des navires spécialement équipés, puis lestées par des matériaux clapés. Deux cavaliers, mis en place par clapage, viennent constituer les pieds des digues, l'espace entre les deux cavaliers étant ensuite rempli de gravier toujours par clapage. Le reste du noyau et les différentes couches de la carapace sont ensuite déposés en place, soit par déversement direct à l'aide de camions, soit par grue. Les briselames sont construits entièrement par moyens maritimes, tous les matériaux étant mis en place par chalands, à l'exception des blocs de carapace pour lesquels il faut recourir à un navire spécial du genre du Lybra utilisé à Rotterdam.

\section{SOLUTIONS 2}

\section{Description}

Les solutions 2 différent de la solution 1 essentiellement par les ouvrages de prise d'eau, les rejets étant identiques. La possibilité d'exécuter ces solutions avec galeries est liée à la qualité de la roche existant sur le site : il est préférable d'avoir des terrains crayeux ou des argiles très compactes, à défaut de roches dures.

Dans la première variante (fig. 7), la tour de prise se présente en plan sous la forme d'un cylindre de section polygonale inscrite dans un cercle de $50 \mathrm{~m}$ de diamètre, avec une hauteur au-dessus du fond variable en fonction de l'implantation (fig. 8). Par exemple, pour un fond à la cote - 10, cette hauteur est de $27 \mathrm{~m}$.

La tour comprend une chambre centrale de $20 \mathrm{~m}$ de diamètre et une chambre annulaire. La chambre centrale se prolonge dans le terrain par un puits qui traverse les couches de sable et de matériaux tendres et pénètre suffisamment dans la roche pour rejoindre les galeries. La chambre centrale est couronnée dans sa partie haute (audessus du fond) par la chambre annulaire qui fait fonction de prise d'eau et dont la paroi extérieure est perforée (du type Jarlan). Le but de cette paroi est d'amortir la houle, et ainsi d'assurer à l'ensemble une meilleure stabilité. 


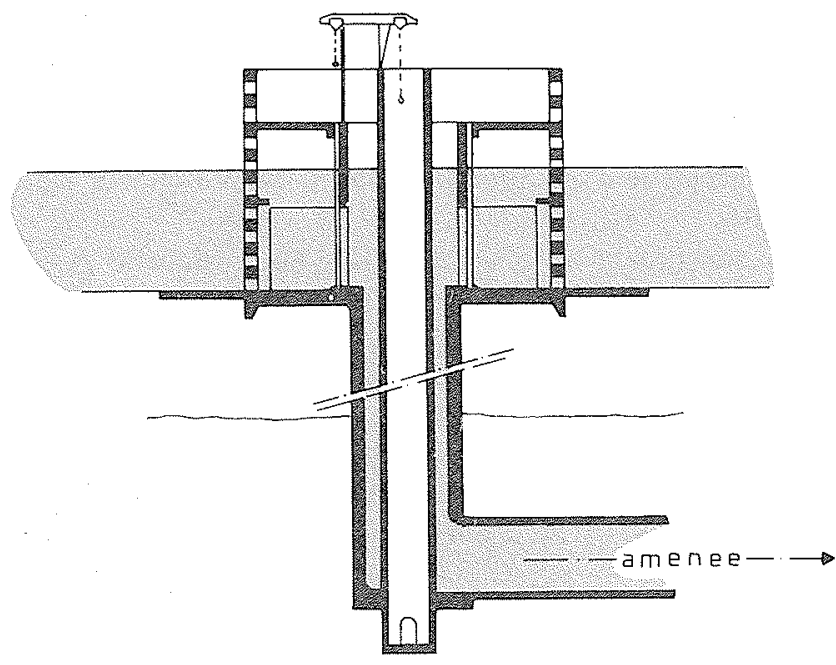

$8 /$ Tour de prise d'eau

La chambre annulaire est pourvue d'un certain nombre de cloisons radiales qui rigidifient l'ensemble et contribuent à tranquilliser l'eau. Les cloisons peuvent être prévues étanches et, en correspondance avec des cloisons similaires dans la chambre centrale, permettent d'établir autant de circuits indépendants qu'il y a de galeries. Le passage de l'eau entre la chambre annulaire et la chambre centrale se fait au travers de grilles destinées à retenir les corps flottants.

Dans laxe de la chambre centrale et du puits la prolongeant, se trouve un puits d'accés du personnel de $3 \mathrm{~m}$ de diamètre, relié sous l'ouvrage à une galerie de petite section venant de terre. La tour représentée permet le raccor- dement de 4 galeries d'eau de $8,50 \mathrm{~m}$ de diamètre. Ces galeries rejoignent la plage et remontent dans un bassin de répartition par un paits en béton de $15 \mathrm{~m}$ de diamètre dépassant du fond du bassin de 1 à $2 \mathrm{~m}$.

Ce bassin est conçu comme le canal d'amenée de la solution 1, avec une largeur de radier de $300 \mathrm{~m}$.

Dans la seconde variante, il y a autant de tours de prise que de galeries; les tours sont identiques à celles de la première variante avec des dimensions réduites de moitié.

\section{Mode d'exécution}

Les tours de prise d'eau pourraient être exécutées en deux phases:

- la partie haute de la tour, c'est-à-dire celle qui se situe au-dessus du fond de la mer, est préfabriquée à terre, en cale sèche, remorquée sur place par flottaison puis coulée par ballastage;

- Ia chambre centrale est alors entièrement remplie de sable, et prolongée jusqu'à la roche sous-jacente par des parois moulées forées dans le sable. Il reste alors à enlever le sable et excaver en puits jusqu'au niveau des futures galeries.

Ces galeries sont forées à partir de la terre ferme; il est d'abord nécessaire d'exécuter un puits de descente du matériel. Le puits est réalisé, par excavation, dans une enceinte en parois moulées prolongée dans la roche, jusqu'au niveau de départ de la conduite.

Cette conduite est entreprise de la façon classique qui avait été choisie pour les galeries du tunnel sous la Manche, avec des voussoirs en béton. Ce type de voussoirs garantit en effet un meilleur écoulement hydraulique que des voussoirs en fonte avec remplissage des alvéoles par du béton. La galerie d'accès est faite suivant la même méthode, mais en plus petit diamètre.

\section{Discussion}

Président: M. J.-C. LeBreton

M. le Président remercie MM. MaINIER et LePETIT pour leur intéressant exposé sur la "superprise " d'eau de la grande centrale de Gravelines et poursuit :

Je vous propose d'engager, dès maintenant, la discussion sur la première partie de l'exposé que nous venons d'entendre; la discussion sur la deuxième partie pourrait être jumelée avec celle de la troisième communication de cette matinée qui traite divers sujets concernant les prises d'eau.

M. ChalenCon (SIMECSOL-Etudes) intervient en ces termes :

Le canal d'amenée d'eau de Gravelines est soumis à la marée et l'amplitude de celle-ci peut, je crois, dépasser $6 \mathrm{~m}$. Par ailleurs, les niveaux d'eau dans les canaux d'alimentation et de rejet qui sont contigus sur une grande longueur, peuvent être assez différents. Compte tenu de la nature du sol - sable fin, de granulométrie comprise entre 100 et $200 \mu$, par suite de perméabilité moyenne - les niveaux d'eau jouent donc un rôle important pour le calcul des ouvrages. Quelles sont les conditions de charge piézométrique prises en compte pour la stabilité :

- des berges inclinées du canal;

- des murs verticaux à terre-plein;

- de l'ouvrage de séparation des canaux.
A propos de l'exposé précédent de M. Duffaut, j'ajouterai que, dans le cas de tunnels débouchant à l'air libre, les têtes posent souvent des problèmes délicats de stabilité de pente; peutêtre sous l'eau ceux-ci ne seraient pas simplifiés.

Enfin, dans la seconde partie de son exposé, M. MaINier a montré, pour une centrale située en bordure de plage, un schéma de circuit d'eau avec entrée d'un côté de la centrale et sortie de l'autre alors qu'à Gravelines, les conditions locales ont conduit à un croisement des circuits d'eau. Ceci implique un passage en siphon...

M. MAINIER répond :

Du fait de la disposition des deux canaux d'amenée et de rejet - situés l'un et l'autre du même côté de la centrale - dont les axes sont parallèles, le franchissement du canal d'amenée par les tuyauteries de rejet n'a pas nécessité un ouvrage exceptionnel; il est réalisé par des tuyaux Bonna franchissant le canal d'amenée de $35 \mathrm{~m}$ de large grâce à des appuis implantés dans le dit canal.

M. Delpuech précise, comme suit, les problèmes posés par la différence de niveau des plans d'eau des deux canaux susvisés :

Les canaux de Gravelines sont adjacents et séparés par des gabionades en palplanches de $12 \mathrm{~m}$ de large, ce qui assure une 
bonne stabilité d'ensemble malgré la différence importante du niveau de l'eau dans ces deux ouvrages.

La paroi du canal d'amenée proprement dit (paroi côté terreplein) est constituée, sur un tiers de sa longueur, par un talus et, sur les deux autres tiers, par une paroi verticale en béton moulé dans le sol. En ce qui concerne le talus, le Port Autonome de Dunkerque a été notre ingénieur-conseil et nous avons adopté des dispositions qui avaient fait leur preuve dans l'avant-port. Pour le calcul des parois moulées, on a admis, derrière celles-ci, un niveau d'eau constant quel que soit le niveau de la mer: les sondages ont, en effet, confirmé que cette cote ne variait que de $6 \mathrm{~cm}$ environ. Le niveau d'eau dans la gabionade oscille au maximum de $15 \mathrm{~cm}$ autour du niveau moyen de la mer $(+3,15 \mathrm{~m}$ : cotes marines).

Pour la Centrale de Gravelines, demande M. LOIRE (TRAMCO S.A.), a-t-on examiné la solution consistant à forer des tunnels d'amenée d'eau dans l'argile des Flandres qui se trouve à la cote -21 ? Cette solution, appliquée par les Anglais de l'autre côté de la Manche, semble d'exécution facile.

Du fait de la proximité du port de Dunkerque dragué à la cote -20 , nous disposions à Gravelines, dit M. MAINIER, de profondeurs suffisantes pour réaliser une prise d'eau en eau calme et la question d'un ouvrage en galerie ne s'est pas posée. Par contre. pour un autre site sur le littoral de la mer du Nord près de Calais, les études s'orientent vers le creusement de tunnels dans l'argile des Flandres.

S'il n'y a pas d'autres questions, conclut M. le Président, en labsence de $M$. LACROIX empêché, je vais donner la parole à $M$. COLLANGE pour l'exposé de la dernière communication de la matinée.

\section{Abstract \\ Water intake and outlet structures of Gravelines power station}

Gravelines power station is situated on the coast just beyond the western end of the new outer harbour. Gravelines itself lies on the North Sea between Dunkirk and Calais. The power station will eventually consist of four $900 \mathrm{MW}$ pressurized-water and four 1,350 MW generating plants. The total once-through cooling-water requirement will be $400 \mathrm{~m}^{3} / \mathrm{s}$. Water inlet and reject structure layout was dictated in large measure by the presence of the new outer harbour. The structures were designed by the Laboratoire National d'Hydraulique in collaboration with the Dunkirk Harbour Authority.

The intake structure was sited at the edge of the outer harbour so that deep water not exposed to large waves would be available close by. Design was based on model tests, the principal objective being to minimize currents in the immediate vicinity of the oil tanker jetties. The outlet structure backs up against the breakwater of the outer harbour and discharges off the beach by the generating plant. Reject at this point minimizes recycle from outlet to inlet but exposes the structure to waves. The outlet structure was also studied on a scale model. The design of the bank protection is similar to that chosen by the Dunkirk Harbour Authority for the new outer harbour, viz. (a) rockfill laid on fascines and polypropylene fabric and $(b)$ superstructures faced with bitumirous concrete. The floor protection will be reinforced or repaired as required by erosion and the commissioning of new plant. Inlet and outlet structures are linked to the power station by canals. The canal floors consist of rockfill. Walls are constituted by rockfill embankment, sheet-piling or concrete.

The second part of the article deals with intakes and outlets located on beaches not comprising any kind of harbour installation. Theoretical studies were undertaken to identify valid designs for the cooling water structures required by power stations near beaches similar to the one at Gravelines, i.e. without breakwaters, etc. Two schemes appeared feasible. The first, based on Gravelines, consists of two "artificial harbours", one each for the intake and outlet. This keeps the structures separate and enables water to be pumped from calm, offshore water. Cooling water in the second scheme is withdrawn via one or more towers a considerable distance out to sea. It is then fed to the power station through an underwater tunnel system. The reject structure is similar to the Gravelines outlet. 